Recepción: 11 / 04 / 2018

Aceptación: 21 / 06 / 2018

(c)

Ciencias de la educación

Publicación: 15 / 09 / 2018

Artículo de investigación

\title{
Incidencia de un programa de lectoescritura en el rendimiento escolar
}

\section{Impact of a literacy program on school performance}

\section{Impacto de um programa de alfabetização no desempenho escolar}

\author{
Marieta Zamora ${ }^{\mathrm{I}}$ \\ marietazamora@hotmail.com \\ Teresa Vargas-Cevallos II \\ teresavargas@hotmail.com \\ Lenin Mendieta-Toledo III \\ lenin.mendietat@ug.edu.ec
}

Correspondencia:_marietazamora@hotmail.com

\footnotetext{
${ }^{\text {I }}$ Docente de la Universidad de Guayaquil, Guayaquil, Ecuador.

${ }^{\text {II }}$ Docente del Instituto Ana Paredes de Alfaro, Guayaquil, Ecuador.

III Magister en Docencia Universitaria e Investigación Educativa, Master Universitario en Actividad Física y Salud, Licenciado en Ciencias de la Educación en la Especialidad de Educación Física, Docente de la Universidad de Guayaquil, Guayaquil, Ecuador.
} 


\section{Resumen}

La lectoescritura es uno de los componentes pedagógicos de mayor influencia en el desarrollo de las funciones cognitivas de los niños, el objetivo del estudio fue determinar los efectos de un programa de lectoescritura en el rendimiento escolar en lengua y literatura en la escuela Padre Antonio Amador de la ciudad de Guayaquil, la muestra estuvo constituida por 32 estudiantes de entre 5 y 12 años que presentaban rezago escolar. Fue un estudio de campo, documental y correlacional; se emplearon como antecedentes las calificaciones en lengua y literatura, entrevista, encuesta y evaluación de la lectoescritura: Test EMLE-T.A.L.E. 2000 antes y después de la aplicación del programa de lectoescritura. Los resultados fueron: en comprensión de los que leen se incrementó un 9\% en muy bueno; en bueno se incrementó un 24\%; en Regulan disminuyó un $17 \%$. En copia de textos simples y compuestos, se incrementó en Muy Bueno un 3\%; Bueno incrementó del 27\%; Regular disminuyó un 24\%. En dictado de cuentos e historias para su redacción, Muy bueno incrementó el 9\%; Bueno incrementó el 15\%; Regular disminuyó el 18\%; en Insuficiente disminuyó el 9\%. En lectura en voz alta de textos simples, Muy bueno incrementó el 21\%; Bueno incrementó el 12\%; Regular disminuyó el 15\%; Insuficiente disminuyó el 30\%. Se concluyó que las intervenciones con programas pedagógicos de refuerzo escolar mejoran sustantivamente el rendimiento escolar en la lectoescritura en los niños.

Palabras claves: programas pedagógicos; lectoescritura; rendimiento escolar.

\section{Abstract}

Literacy is one of the pedagogical components of higher influence in the development of the cognitive functions in children, the aim of this research is to determine the effects of a literacy program in school performance at language and literature in the school Padre Antonio Amador of Guayaquil, the set sample was 32 students between 5 and 12 years' old who presented school lag. It was a field research, documentary, and correlational; it was used as background the scores of language and literature, interview, survey, and literacy evaluation: EMLE-T.A.L.E. 2000 Test before and after the application of the literacy program. Tue results were: in comprehension of the ones who read increased $9 \%$ in very good; in good increased $24 \%$, in average decreased $17 \%$. In transcription of simple and complex texts, it increased in very good 3\%; good increased $27 \%$; average decreased $24 \%$. In dictation of tales and stories for drafting, very good increased $9 \%$; 
good increased 15\%; average decreased 18\%; in inadecuate decreased 9\%. In reading out loud of simple texts, very good increased $21 \%$; good increased $12 \%$; average decreased $15 \%$; inadecuate decreased 30\%. The conclusion was that interventions with pedagogical school reinforcement programs improve substantively the school performance in children literacy.

Keywords: pedagogical programs; literacy; school performance.

\section{Resumo}

A alfabetização é um dos componentes educacionais de maior influência sobre o desenvolvimento das funções cognitivas das crianças, o objetivo do estudo foi determinar os efeitos de um programa de alfabetização no desempenho escolar em artes da linguagem na escola Padre Antonio Amador Na cidade de Guayaquil, a amostra foi constituída por 32 alunos entre 5 e 12 anos que apresentavam carteira escolar. Foi um estudo de campo, documentário e correlacional; eles foram usados como notas de fundo em língua e literatura, entrevista, exame e avaliação da alfabetização: Teste Emle- T.A.L.E. 2000 antes e depois da aplicação do programa de leitura e escrita. Os resultados foram: na compreensão de quem leu, aumentou 9\% em muito bom; em bom aumentou 24\%; em Regulan diminuiu 17\%. Em cópia de textos simples e compostos, foi aumentado em 3\% em Muito Bom; Bom aumento em 27\%; regular diminuiu em 24\%. No ditado de histórias e histórias para a sua escrita, muito bom aumentou 9\%; Bueno aumentou 15\%; regular diminuiu 18\%; em Insuficiente, diminuiu 9\%. Em ler em voz alta de textos simples, muito bom aumentou 21\%; Bueno aumentou 12\%; regular diminuiu 15\%; insuficiente diminuiu 30\%. Concluiu-se que as intervenções com programas educacionais de apoio escolar melhorar substancialmente o desempenho dos alunos na alfabetização em crianças.

Palavras chave: programas pedagógicos; alfabetização; rendimento escolar.

\section{Introducción}

El lenguaje desde el punto de vista funcional es una de las actividades de socialización más complejas y la única que permite la comunicación a través de señales escritas -lenguaje escrito-, o verbales -lenguaje oral-, es a través del lenguaje que se produce la trasferencia del conocimiento de generación en generación. señalan que el lenguaje se concibe como "una actividad humana compleja que asegura las funciones básicas de comunicación y representación, estas funciones no 
se excluyen mutuamente, sino que son simultáneas y complementarias en la actividad lingüística de cada uno de los hablantes" (Avendaño \& Miretti, 2006, p. 15).

La escritura es considerada como el mayor invento de la humanidad, a través de esta, el ser humano ha dejado plasmada su historia -antes de la escritura se plasmó en diferentes formas que se podría considerar escritura no normalizada-, la escritura a pesar de parecer una actividad natural y espontanea a la que todos los individuos logramos llegar, es sin temor a equivocaciones una destreza lingüística muy difícil de adquirir. Esta dificultad se ahonda más y de forma trasversal a la hora de producir oraciones, párrafos, historias y más; se puede evidenciar la sensación de inseguridad que reflejan muchos estudiantes e incluso jóvenes de todos los niveles sociales cuando se les solicita una actividad que requiera la competencia escrita

La lectura es el acto de interpretar y decodificar mediante la vista, el valor fónico de una suma secuenciada de signos escritos llamados letras, números o símbolos y reproducirlos en la mente en forma de escenas pseudo-vivenciales. Después de esto y dependiendo del interés por la lectura se guardan en el subconsciente para ser utilizados cuando sea necesario. Sobre la lectura se puede decir mucho, en con su obra permite comprender que la lectura en un proceso complejo en donde juegan roles diferentes e interrelacionados a la vez el pensamiento y el lenguaje que realizan diferentes transacciones, cuando el lector trata de dar sentido a una frase o párrafo, emite desde su cerebro una serie de preguntas y respuestas subconscientes producto de su acervo intelectual que atan y desatan lo que el autor quiere dar a entender o el lector entiende

Uno de los principios fundamentales de los países a nivel mundial, es otorgar una educación igualitaria y de calidad a sus habitantes a lo largo de toda su vida, la organización para la educación en el mundo (UNESCO, 2001) indica "Recibir una educación de calidad a lo largo de toda la vida es un derecho congénito de cada niño, mujer u hombre", a pesar de los múltiples esfuerzos de corte teóricos que realiza esta organización encargada de velar por una educación igualitaria en el mundo, esta no llega por igual y en los mismos niveles de calidad. Existe una discriminación de parte de los gobiernos a la hora de brindar ese servicio a sus habitantes.

Según datos de la organización mencionada, en el mundo más de 1500 millones de jóvenes y niños reciben educación (pág. 7) en el sistema regular de enseñanza de los distintos niveles 
educativos, 52 millones de niños se han incorporado a la educación primaria, sin embargo, según la misma fuente más de 67 millones de infantes siguen sin escolarizarse. Un dato preocupante es la deserción escolar. En el tema que nos compete para el análisis y propuesta es la que en el mundo más del $17 \%$ de personas adultas en este estudio -el estudio de las autoras en con niñoses decir unos 793 millones de las cuales 528,5 millones no poseen competencias básicas de lectura y escritura.

La lectoescritura es la suma de la escritura y lectura, es una actividad intelectual que demanda de la psicomotricidad (pensamiento y acción motora), se constituye en una habilidad que se fortalece como destreza y llega a convertirse en una competencia para la vida de las personas, es esencial para el desenvolvimiento en la sociedad del conocimiento en que se vive, de allí su gran importancia es uno de los componentes pedagógicos de mayor influencia en el desarrollo de las funciones cognitivas de los niños y con ellas del rendimiento escolar.

Los programas pedagógicos son diseños metodológicamente estructurados que permiten orientar a los docentes en los procesos de enseñanza. Es decir, es la forma de desarrollar las actividades de enseñanza y os objetivos a cumplir. Los programas pedagógicos pueden tener diferentes estructuras dependiendo del nivel de enseñanza, del grupo etario, de las características particulares del grupo y del fin propuesto. La secuencia metodológica para diseñar un programa es: planteamiento del problema, marco teórico del objeto de conocimiento, diseño de una evaluación diagnóstica -instrumentos de evaluación- (aquí se incluye el análisis e interpretación de la evaluación), diseño y ejecución del programa, evaluación post programa, análisis e interpretación de los resultados, informe de la finalización del programa y divulgación.

Un programa educativo debe fundar su estructura en principios, teorías y leyes del país en donde se va a realizar la intervención y lleva niveles de concreción: nivel nacional -principios, objetivos y fines de la educación de un país-, nivel zonal -los países se encuentran divididos en zonas, regiones o estados dentro del estado-, nivel local -el institucional, el último nivel es el aula de clase con los sujetos cognoscentes. 
Desde el gobierno ecuatoriano se han diseñado programas curriculares que tiene por objetivo común el desarrollo holístico del ser humano y con ello cumplir con el mandato constitucional que reza en el artículo 27 de la carta magna ecuatoriana. Asamblea Nacional del Ecuador, (2011) indica:

La educación se centrará en el ser humano y garantizará su desarrollo holístico, en el marco del respeto a los derechos humanos, al medio ambiente sustentable y a la democracia; será participativa, obligatoria, intercultural, democrática, incluyente y diversa, de calidad y calidez; impulsará la equidad de género, la justicia, la solidaridad y la paz; estimulará el sentido crítico, el arte y la cultura física, la iniciativa individual y comunitaria, y el desarrollo de competencias y capacidades para crear y trabajar. La educación es indispensable para el conocimiento, el ejercicio de los derechos y la construcción de un país soberano, y constituye un eje estratégico para el desarrollo nacional. (p. 27, 28).

Rendimiento escolar es el nivel cuantitativo del conocimiento adquirido por los estudiantes, este se ve reflejado en los resultados de las evaluaciones o exámenes que se aplican a los estudiantes en el trascurso del año escolar. El hombre desde su infancia trata de destacar entre sus pares y ser reconocido por sus logros, sin embargo, para que el estudiante aprenda y tenga un buen rendimiento, Cartagena, M. (2008), citado en Galleguillos Herrera \& Olmedo Moreno (2017) señala que es requisito sine qua non que este sea cognitivamente capaz de afrontar y cumplir las tareas de aprendizaje y además, que se encuentre motivado a adquirir el aprendizaje y cumplir las tareas. Citado en

Estudios recientes (Castro Torres, M. F. D. P. 2016; Magaña, M., \& Ruiz-Lázaro, P. 2015; Londoño-Muñoz, N., Jiménez-Jiménez, S., González-Alexander, D. C., \& Solovieva, Y. 2016) dan cuenta de la importancia de las intervenciones pedagógicas en estudiantes con bajo rendimiento escolar, en el caso específico de este estudio de la lectoescritura. Explayando más el tema del rendimiento escolar se acude a Magaña, M., \& Ruiz-Lázaro, P. (2015, p. 2), quienes indican que "Un niño o adolescente presenta "problemas escolares" cuando sus resultados pedagógicos están por debajo de sus capacidades intelectuales". 


\section{Metodología}

Fue un estudio de campo, documental y correlacional. La muestra estuvo constituida por 32 estudiantes de la escuela Padre Antonio Amador de la ciudad de Guayaquil. Fue un estudio no aleatorio y se consideró a los estudiantes que presentaban bajo rendimiento en lengua y literatura, los niños tenían entre 7 y doce años, pertenecían al grupo de estudiantes que llevaban un rezago escolar por factores ajenos al estudio, sin embargo, se utilizó estos antecedentes para la formulación del problema. Se obtuvo las calificaciones de la asignatura de lengua y literatura de los estudiantes del año escolar anterior y las del presente año -luego del programa-, se realizó una entrevista con el director y gerente de la institución educativa para determinar las causas intrínsecas y extrínsecas a las que se atribuía el bajo rendimiento escolar en los niños, se encuestó a los docentes que impartían la asignatura y se evaluó a los estudiantes con la prueba de lectoescritura ya preestablecida: Test EMLE-T.A.L.E. 2000 antes y después de la aplicación del programa de lectoescritura.

El programa de intervención se fundó en la técnica ERCA, la misma se caracteriza por optimizar el proceso de aprendizaje y tiene como base el trabajo dirigido a la solución de problemas, los pasos de esta técnica son: experiencia o requisitos previos, reflexión, conocimiento información, acción o aplicación. El programa tuvo una duración de 16 semanas y 30 minutos cada actividad, se trabajó en la primera hora de la mañana y en la primera hora después del receso o recreo es decir dos actividades diarias, dando un total de 160, cuenta con un sólido componente pedagógico, para que todos puedan conocer el programa integro, este se puede descargar a través del siguiente link: https://es.slideshare.net/leninmendietatoledo/programa-de-lectoescrituradesde-la-gestin-social-del-conocimiento.

\section{Resultados}

Entrevista. A directivos de la institución, director (D) y gerente (G) de la fundación, estos contestaron a las siguientes preguntas:

Entrevistador (E)

¿Considera Usted que la institución presenta falencias en cuanto a la ejecución de estrategias para el fortalecimiento de la lectoescritura en los estudiantes? 
D: la institución realiza lo que está en sus manos, no se puede hacer más, tenemos un limitado número de docentes, los cuales por las características particulares que tiene los estudiantes, no pueden realizar tareas que se constituyan en la solución a la problemática de la lecto-escritura.

G: De parte de la fundación se ha conseguido realizar algunas acciones con la Universidad de Guayaquil, lamentablemente el convenio está caducado y eso limita la intervención de los programas educativos que plantea la academia.

E: En el PEI de la institución tienen como meta el rediseñar y actualizar los módulos de la escuela a los nuevos esquemas de aprendizaje, por medio de capacitaciones y planificaciones, ¿cuál considera usted que es la acción más pertinente de parte de la Universidad para coadyubar a los procesos que la institución educativa quiere implementar?

D: De lo que se trata es de lograr confrontar temas y destrezas aplicables a nuestra realidad, esto se lo conseguirá si de parte de la Universidad se brinda el apoyo pedagógico a través de planificaciones pertinentes al objeto del problema, eso sería llegar a la meta.

G: De la realidad institucional se tiene que para noviembre de este año se tendría que tener listo el rediseño de los módulos, pero esto no se ha cumplido pues no existió el diseño modular de las asignaturas.

E: Usted considera que la Universidad de Guayaquil ¿puede gestionar proyectos de consultoría y apoyo pedagógico para la escuela?

D: Efectivamente ese es el anhelo de parte de la institución, el que la universidad siga aportando desde la cooperación desinteresada con el diseño y ejecución de proyectos encaminados a fortalecer las competencias de los estudiantes y menguar sus flaquezas o debilidades, en esta ocasión se tiene dos grandes problemas: la deserción escolar y el bajo rendimiento en lengua y literatura

G: la gestión de la academia debe trabajarse para que el trasvase del conocimiento llegue a la sociedad, es una buena forma de cumplir el encargo social que es generar producción desde una visión responsable y humanista.

E: ¿Considera Usted que hay una relación entre los procesos pedagógicos que genera el docente y el rendimiento escolar de los estudiantes?

D: El docente es el responsable directo del éxito o fracaso de los estudiantes, en este caso en particular se puede eximir a los maestros de esa falencia que se presenta en cuanto a la lectoescritura, pues es muy diferente el tratamiento que se debe dar y manejar con los niños de este 
centro de educación, son niños que vienen arrastrando un lastre social muy pesado para ellos, la pobreza y el entorno social en que habitan les hace ser vulnerables a muchas situaciones que les trae como consecuencia primero la deserción escolar y segundo que los procesos de enseñanzaaprendizaje no llegan a ellos de manera regular, es por ello lo del sistema modular de enseñanza y los programas de refuerzo escolar que desde ustedes les llega a los niños

G: La relación se produce desde dos vectores relacionales: primero es que los programas se deben diseñar en relación con el contexto educativo, siguiendo las pautas del ministerio, pero marcando las diferencias particulares desde lo social y económica y, segundo que las estrategias de enseñanza-aprendizaje deben estar cargadas de componentes motivacionales para llegar a los niños y que estos no se aburran, además del componente llamado amor. Esas son las claves para que estos niños aprendan y lo hagan bien. El tercer componente que no lo menciono por considerarlo que ésta implícito es la competencia pedagógica de los docentes.

E: ¿Que debe llevar un programa de enseñanza-aprendizaje en este grupo estudiantil?

D: Debe llevar el juego como base de la enseñanza, son niños que trabajan muchas horas ayudando a sus padres y no tienen la oportunidad de ser lo que su naturaleza les pide a gritos (ser niños), un programa debe llevar en todas sus clases el juego.

G: considero que, si se programan clases basadas en la lúdica, estos niños aprenderán de mejor manera, se sentirán a gusto, otro punto a tomar en cuenta es el acercamiento del docente como el amigo no con la pared que el profesor normal coloca a sus estudiantes, acá es diferente, fundación salesiana basa su filosofía de enseñanza en el amor, en la amistad y desde allí se llega al conocimiento.

Análisis e interpretación de las respuestas.

Desde un análisis hermenéutico se considera que tanto director como gerente de la institución se encuentran inconformes con el statu quo académico de la institución, al ser esta una fundación sin fines de lucro, encaminada a la trasformación del ser humano en persona humana desde una filosofía salesiana al servicio de la humanidad. Instan a desterrar la pobreza y la balcanización de la educación y a cubrir la brecha en las dimensiones: humanística, educativa, política, económica y social, invitan a des-invisibilizar al sujeto objetivado y cosificado por la sociedad y los gobiernos, y exigen comedidamente que la Universidad de Guayaquil se haga presente en el campo en el objeto para tratar de presentar y ejecutar más proyectos que logren paliar estas desigualdades. 
El limitado número de docentes hace que los esfuerzos sean pocos al lado de la problemática educativa, en este caso la lecto-escritura; invitan a la Universidad de Guayaquil a presentar como alternativa planificaciones como la que se llevó a cabo en los meses de estudio y cargan con la culpa del bajo rendimiento de los estudiantes por la falta de docentes y al tratamiento inapropiado que se les da a los estudiantes -recordar que son niños con rezago escolar-; terminan manifestando que es a través del juego que los niños logran aprender de mejor manera, pues estos han padecido de lo que se denomina pobreza lúdica -falta de juego en la edad infantil-, producto de la pobreza en que viven y que tienen que salir a trabajar.

Gráfico 1. Encuesta a los docentes

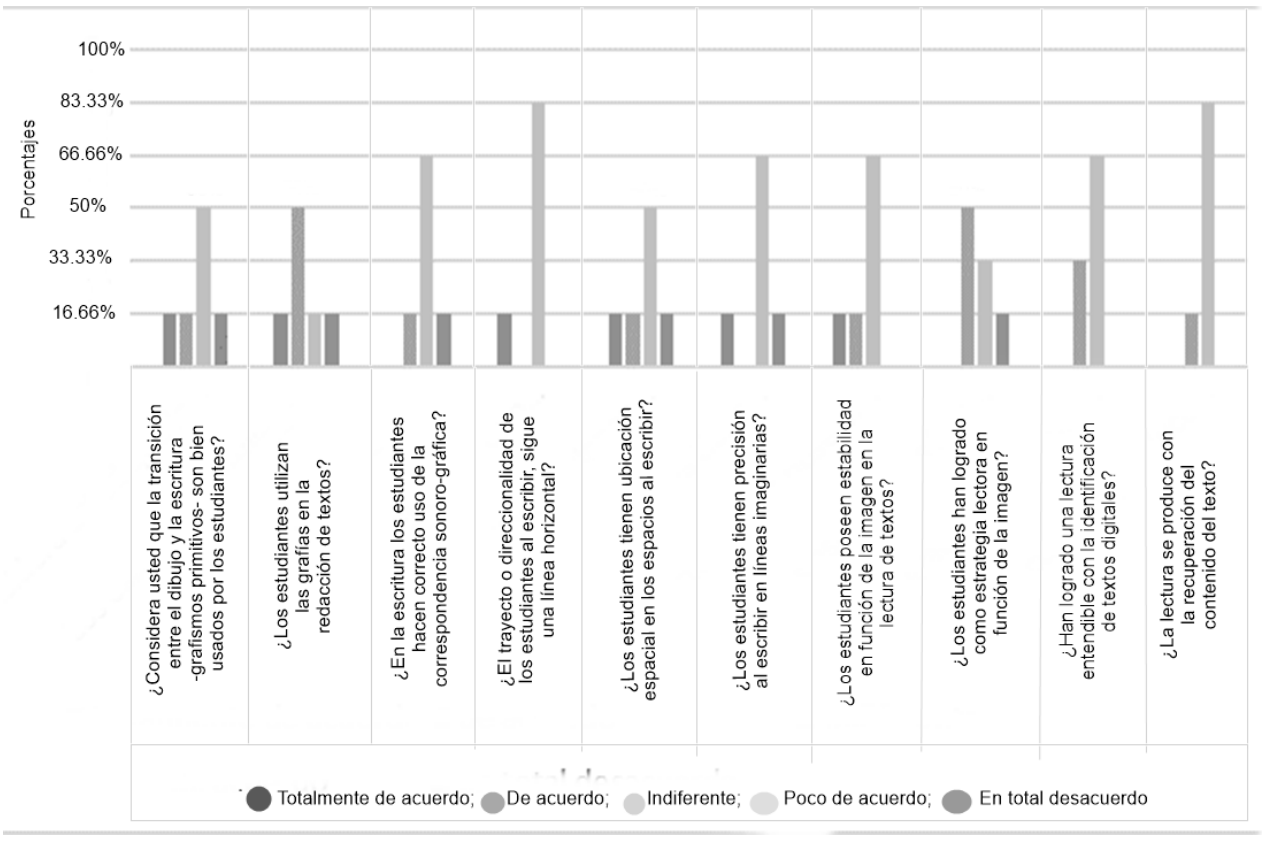

Fuente: Elaboración propia 


\section{Gráfico 2. Escritura}

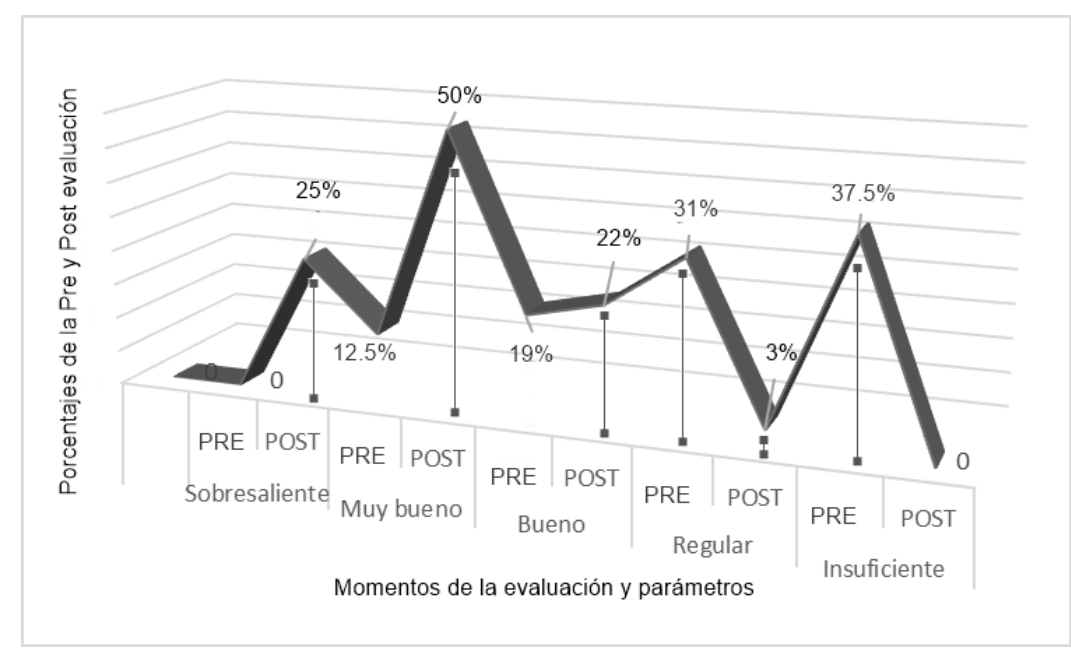

Fuente: Elaboración propia

Análisis. En la escritura se evidencia un incremento del $25 \%$ en la valoración de sobresaliente; en muy bueno pasan de $12.5 \%$ a $50 \%$, es decir un incremento del $37.5 \%$; en la valoración bueno pasan de $19 \%$ a $22 \%$ es decir un incremento del $3 \%$; se reduce el porcentaje de estudiantes con valoración regular de $31 \%$ a $3 \%$ es decir decrece un 28\%; antes del programa existió un 37.5\% de insuficiente, después del mismo un $0 \%$.

\section{Gráfico 3. Lectura}

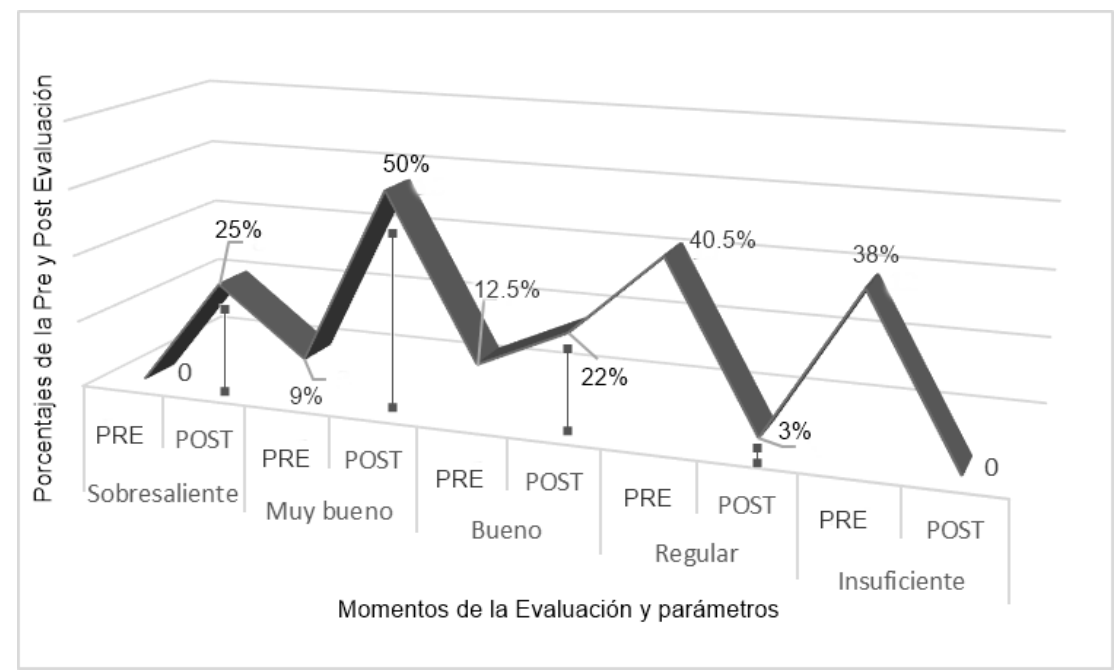

Fuente: Elaboración propia 
Análisis. En la lectura se evidencia un incremento del $25 \%$ en la valoración de sobresaliente, en la pre evaluación había un $0 \%$ de sobresalientes; en muy bueno pasan de $9 \%$ a $50 \%$, es decir un incremento del $41 \%$; en la valoración bueno pasan de $12.5 \%$ a $22 \%$ es decir un incremento del 9.5\%\%; se reduce el porcentaje de estudiantes con valoración regular de $40.5 \%$ a $3 \%$ es decir decrece un 37.5\%; antes del programa existió un 38\% de insuficiente, después del programa un $0 \%$.

Gráfico 4. Test EMLE-T.A.L.E. 2000

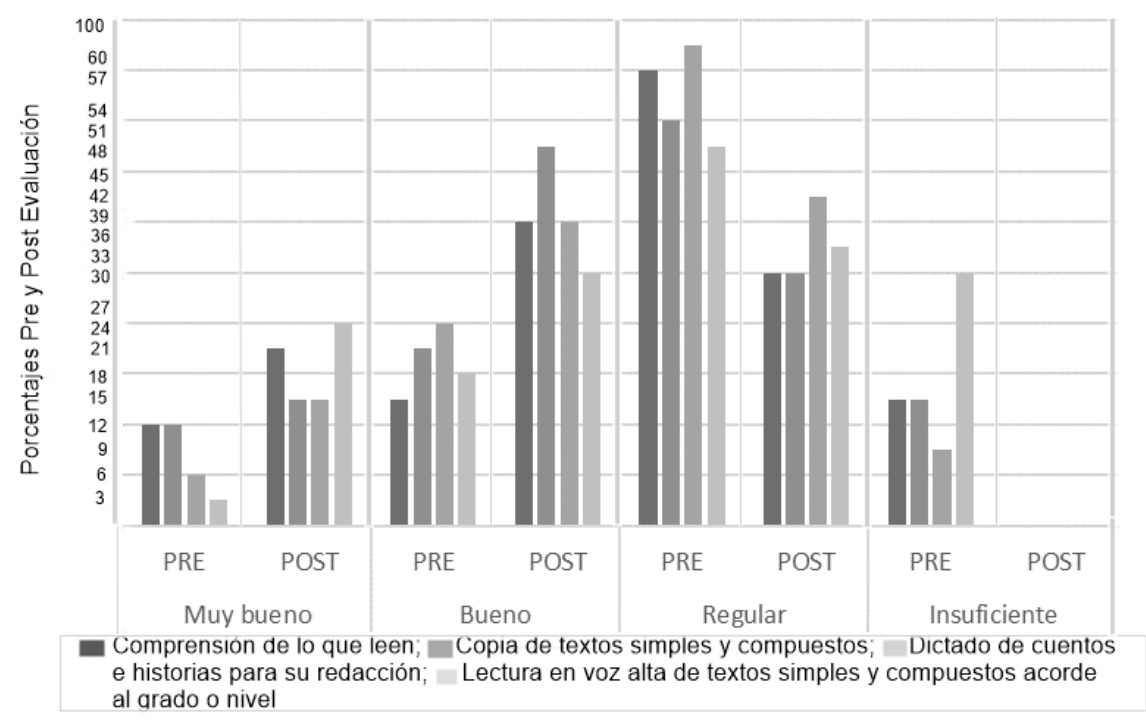

Fuente: Elaboración propia

Análisis. En la prueba TALE los estudiantes en comprensión de lo que leen, en la valoración Muy buena se incrementó un 9\% (pasan del 12\% al 21\%); en Bueno pasan del 15\% al 39\%; en Regulan disminuyó del 57\% al 30\%; en Insuficiente en el post disminuyó del 15\% a 0\%. En la copia de textos simples y compuestos, en la valoración de Muy Bueno se incrementó del $12 \%$ al $15 \%$ el número de estudiantes; en Bueno se incrementó del 21\% al 48\%; en Regular disminuyó del $54 \%$ al 30\%; en Insuficiente disminuyó del 15\% a $0 \%$. En el dictado de cuentos e historias para su redacción en Muy bueno se incrementó del 6\% al 15\%; en Bueno se incrementó del 24\% al 39\%; en Regular disminuyó del 60\% al 42\%; en Insuficiente disminuyó del 9\% al 0\%. En la lectura en voz alta de textos simples y compuestos acorde al grado o nivel en Muy bueno se incrementó del 3\% al 24\%; en Bueno se incrementó del 18\% al 30\%; en Regular disminuyó del $48 \%$ al 33\%; en Insuficiente disminuyó del 30\% al 0\%. 


\section{Discusión}

Es incuestionable el beneficio que acarrea consigo el trabajar programas pedagógicos de lectoescritura en niños con rezago escolar, siendo el lenguaje una de las actividades más complejas y a la vez la única que permite la socialización de los seres vivos, están se comportan solapadamente lectura y escritura en aras de la comunicación como parte de la actividad humana, coincidiendo con (Avendaño \& Miretti, 2006, p. 15).

La escritura se constituye una destreza y como tal hay que entrenarla adecuadamente siendo la pedagogía la autorizada a desarrollar esta difícil destreza lingüística (Sotomayor, Molina, Percy, \& Hernández, 2013), de igual forma la lectura es un proceso complejo de razonamiento y lenguaje que requiere del cerebro para preguntar y contestar, es decir interpretar los contenidos de las lecturas, dependiendo las respuestas del nivel o acervo intelectual del lector (Goodman, 1991).

La lectoescritura demanda de pensamiento y acción motriz, es decir es una secuencia intelectomotora que se constituye en una competencia, luego de pasar por las fases de habilidad y destreza entrenadas, su importancia estiba en la apertura a la sociedad del conocimiento a través de esta y que además ayuda y en gran medida al rendimiento escolar (Mendoza \& Rojas, 2016).

Si se adquieren estas competencias y el estudiante afronta y cumple las tareas desde una motivación intrínseca o extrínseca sobre el objeto del conocimiento, de acuerdo a Cartagena, M. (2008), citado en Galleguillos Herrera \& Olmedo Moreno (2017) se logrará entonces mejorar el rendimiento escolar. Se coincide con los estudios de (Castro Torres, M. F. D. P. 2016; Magaña, M., \& Ruiz-Lázaro, P. 2015; Londoño-Muñoz, N., Jiménez-Jiménez, S., González-Alexander, D. C., \& Solovieva, Y. 2016) sobre la ventaja de intervenir a los estudiantes que presentan bajo rendimiento escolar mediante programas de lectoescritura

\section{Conclusiones}

Las teorías y estudios de intervención pedagógica se constituyen en el mecanismo idóneo para lograr mejorar el rendimiento escolar en los estudiantes, así se cumple el objetivo de una educación igualitaria y de calidad para las personas y que les servirá en su vida, tal como lo 
indica la UNESCO, (2001). Se cumple en parte y en lo que corresponde con el artículo 27 de la carta magna ecuatoriana que en síntesis exige una educación holística para toda la vida; se cumple con la misión de la Universidad de Guayaquil en realizar el trasvase del conocimiento científico hacia la sociedad y es a través de la educación que se logra esta misión enmarcada en el Buen Vivir de los ecuatorianos como filosofía de vida.

Por tanto, se concluye que un programa de lectoescritura influye positivamente en el rendimiento escolar en la signatura de lengua y literatura en estudiantes que presentan rezago escolar; pues en la línea de impulsar proyectos de innovación para el mejoramiento de la calidad educativa, se puede dar inició a la construcción de un programa educativo que busque fortalecer, el proceso de aprendizaje de la lectura comprensiva y el desarrollo de los valores humanos. De allí que un programa de lectoescritura, pueda presentar como objetivos principales desarrollar la competencia lectora en las niñas y los niños, y promover de manera sistemática el aprendizaje de valores.

\section{Referencias Bibliográficas}

Asamblea Nacional del Ecuador. (7 de mayo de 2011). Constitución de 2008 - Asamblea Nacional del Ecuador, electrónica. Recuperado el 4 de julio de 2017, de Constitución de 2008 Asamblea Nacional

del

Ecuador: http://www.asambleanacional.gob.ec/sites/default/files/documents/old/constitucion_de_bolsillo.p df

Avendaño, F., \& Miretti, M. L. (2006). El desarrollo de la lengua oral en el aula. Rosario, Mendoza, Argentina: HomoSapiens Ediciones. doi: ISBN. 10:950-808-492-7

Galleguillos Herrera, P., \& Olmedo Moreno., E. (2017). Autoeficacia académica y rendimiento escolar: un estudio metodológico y correlacional en escolares. REIDOCREA, 6(14), 156-169. doi:2254-5883

Goodman, K. (1991). El proceso de lectura: consideraciones a través de la lengua y el desarrollo. En E. Ferreriro, \& M. G. Palacio, Nuevas perspectivas sobre los procesos de lectura y escritura (pág. 13). México, México: Siglo XXI. doi: ISBN: 968-23-1600-6

Mendieta, L., Mendieta, R., \& Vargas, T. (2017). Psicomotricidad Infantil. Guayaquil, Guayas, Ecuador: CIDE. doi: ISBN: 978-9942-759-28-3 
Mendoza, M., \& Rojas, R. (2016). Resultados concluyentes del estudio sobre el nivel de lectoescritura en cadetes de la Escuela Militar (2010-2014). General José María Córdova, 14(18), 346-360. doi: http://dx.doi.org/10.21830/19006586.93

Sotomayor, C., Molina, D., Percy, B., \& Hernández, C. (2013). Caracterización de problemas ortográficos recurrentes en alumnos de escuelas municipales chilenas de $3^{\circ}, 5^{\circ}$ y $7^{\circ}$ básico. Revista signos, 105-131. doi: ISNN: 0718-0934; http://dx.doi.org/10.4067/S071809342013000100005

UNESCO. (13 de SEPTIEMBRE de 2001). La UNESCO y la educación: toda persona tiene derecho a ... - unesdoc. Obtenido de La UNESCO y la educación: toda persona tiene derecho a ... - unesdoc: http://unesdoc.unesco.org/images/0021/002127/212715s.pdf. 